\title{
MANIFOLDS WITHOUT CONJUGATE POINTS
}

\author{
BY \\ MARSTON MORSE AND GUSTAV A. HEDLUND
}

1. Introduction. If a closed two-dimensional Riemannian manifold $M$ is homeomorphic to a sphere or to the projective plane and $A$ is any point of $M$, there exists a geodesic passing through $A$ with a point on it conjugate to $A$ (cf., e.g., Myers [1, p. 48, Corollary 2$]$ ) $\left({ }^{1}\right)$. There exists a closed two-dimensional Riemannian manifold of any other given topological type such that no geodesic on the manifold has on it two mutually conjugate points. The simplest examples of these are manifolds of vanishing Gaussian curvature in the case of the torus and the Klein bottle, and manifolds of constant negative curvature in the remaining cases. In all these particular examples the differential equations defining the geodesics can be integrated and the properties in the large of the geodesics can be determined. In the case of the flat torus or flat Klein bottle the geodesics are either periodic or recurrent but not periodic. In the case of closed manifolds of constant negative curvature the behavior of the geodesics is much more complex, but among other types there exist transitive geodesics.

In this paper our starting point will be the assumption that we have a closed two-dimensional Riemannian manifold $M$ such that no geodesic on $M$ has on it two mutually conjugate points. Our aim is to determine what properties of $M$ or of the geodesics on $M$ must follow from this hypothesis. The possibility that $M$ be homeomorphic to the sphere or to the projective plane is thereby eliminated and it is necessary to divide the possible closed manifolds in to two classes. The first class is made up of manifolds homeomorphic to the torus or to the Klein bottle; the second class is made up of the remaining possible manifolds.

In Part I we study manifolds of the first class. Here the universal covering surface of $M$ is a plane $\Theta$ provided with a metric which satisfies certain group properties. It is shown that the hypothesis that there are no two mutually conjugate points on any geodesic implies that the geodesics in $\Theta$ behave in numerous respects like straight lines. Each unending geodesic $g$ is the topological image of a straight line. There exists a constant $R$ determined by $M$ such that any unending geodesic lies between two parallel straight lines at a distance apart not exceeding $R$. Two geodesics can intersect in at most one point. A parallelism property holds in that, if $g$ is any unending geodesic and $P$ is any point not on $g$, there exists exactly one geodesic passing through $P$ and not intersecting $g$.

Presented to the Society, September 5, 1941; received by the editors February 11, 1941.

(1) References will be found in the bibliography at the end of the paper. 
There is the possibility that the hypothesis concerning the nonexistence of conjugate points implies that the Gaussian curvature vanishes identically. The authors have not been able to verify or disprove this conjecture.

However, it will be shown that an additional hypothesis concerning the nonexistence of focal points is sufficient to insure the identical vanishing of the Gaussian curvature.

This additional hypothesis as well as that concerning the nonexistence of conjugate points is implied by the hypothesis that, if $g$ is any geodesic in $\Theta$ and $P$ is any point not on $g$, there is just one geodesic passing through $P$ and orthogonal to $g$.

In Part II we consider manifolds of the second class, as well as certain manifolds which are not closed. Here the universal covering surface is the interior of the unit circle. The hypothesis that no geodesic have on it two mutually conjugate points is fulfilled by a large class of manifolds and, in particular, by those with everywhere negative curvature.

The question with which we shall be principally concerned is that of the existence of transitive geodesics. The existence of such geodesics on closed orientable manifolds of genus greater than one has been proved under various hypotheses. The first proof which did not assume that the Gaussian curvature of the manifold was everywhere negative was given by Morse (cf. Morse [2]), who showed the existence of transitive geodesics under the hypothesis of uniform instability. Subsequently, Hedlund (cf. Hedlund [2]) proved that such geodesics exist on an extended class of manifolds, provided a different condition, which we term ray instability, is fulfilled. Both uniform instability and ray instability imply that no geodesic has on it two mutually conjugate points.

In the present paper we show that for a large class of two-dimensional manifolds the hypothesis that no geodesic has on it two mutually conjugate points is sufficient to imply the existence of transitive geodesics.

The attainment of the new results of both parts of the paper is made possible by use of the well known recurrence theorem of Poincaré which states that in a dynamical system with a bounded invariant integral almost all the motions are stable in the sense of Poisson.

Part I. Manifolds of eUclidean type

2. A class of simply-connected Riemannian manifolds of euclidean type. We consider the quadratic form

$$
d s^{2}=F^{2}(x, y)\left(d x^{2}+d y^{2}\right)
$$

where $F(x, y)$ is of class $C^{3}$ and

$$
0<a \leqq F(x, y) \leqq b,
$$

in the $(x, y)$-plane $\Theta$. This two-dimensional Riemannian manifold will be denoted by $M(F)$ and termed a manifold of euclidean type. 
The length of a rectifiable curve $\gamma$ on $M(F)$ will be its length as determined by the metric (2.1) and will be denoted by $L(\gamma)$. The geodesics corresponding to (2.1) are of class $C^{2}$ in terms of the arc length as parameter and in terms of suitably chosen parameters giving the initial conditions.

The manifold $M(F)$ is complete in the sense of Hopf and Rinow (cf. Hopf and Rinow [1]) and corresponding to a given pair of points $P$ and $Q$ of $\Theta$ there exists a geodesic segment joining $P$ and $Q$ which affords an absolute minimum of length relative to all rectifiable curves joining $P$ and $Q$. Following Morse (cf. Morse [1]) we term such a geodesic segment a geodesic segment of class $A$. We term the length of a class $A$ geodesic segment joining $P$ and $Q$ the distance between $P$ and $Q$ and denote it by $D(P, Q)$. It is easily shown that the metric $D(P, Q)$ satisfies the usual conditions (cf. Hausdorff $[1, \mathrm{p} .94]$ ) which are imposed on a metric.

A geodesic segment of class $A$ is necessarily a simple curve. For, if the point $P$ were a multiple point of the segment, we would have a geodesic segment of class $A$ with identical end points and such a segment obviously could not be of class $A$.

A geodesic ray $r$ is a geodesic with an initial point $P$ which in terms of its arc length $s$ measured from $P$ is the continuous image of the half-line, $0 \leqq s<\infty$. A geodesic ray is of class $A$, if every finite segment of it is a class $A$ geodesic segment. It is evident that a geodesic ray of class $A$ can have no multiple points and is the topological image of a half-line. As to the existence of class $A$ geodesic rays, it is known (cf. Rinow [1]) that, if $P$ is any point of $\Theta$, there exists a class $A$ geodesic ray with initial point $P$.

An unending geodesic is a geodesic which in terms of its arc length $s$ is the continuous image of the whole $s$-axis, $-\infty<s<\infty$. An unending geodesic $g$ is said to be of class $A$ if each finite segment of $g$ is of class $A$. A class $A$ unending geodesic can have no multiple points and is the topological image of a straight line. Under the conditions thus far imposed on $M(F)$ we cannot infer the existence of unending geodesics of class $A$ on $M(F)$. As a matter of fact, it is not difficult to construct manifolds $M(F)$ on which there are no unending geodesics of class $A$.

If we denote the euclidean distance (i.e., the distance when $F(x, y) \equiv 1$ ) between $P$ and $Q$ by $E(P, Q)$, the following lemma can be stated.

Lemma 2.1. If $P$ and $Q$ are arbitrary points of $\Theta$,

$$
a E(P, Q) \leqq D(P, Q) \leqq b E(P, Q) .
$$

For let $\gamma$ be a class $A$ geodesic segment joining $P$ and $Q$. With the aid of (2.2) we find that

$$
D(P, Q)=\int_{\gamma} F(\dot{x}+\dot{y})^{1 / 2} d t \geqq a \int_{\gamma}\left(\dot{x}^{2}+\dot{y}^{2}\right)^{1 / 2} d t \geqq a E(P, Q),
$$


and the first inequality of the lemma is proved. If we let $\lambda$ be the $E$-line (euclidean line) segment joining $P$ and $Q$, we have

$$
D(P, Q)=\int_{\gamma} d s \leqq \int_{\lambda} d s \leqq b \int_{\lambda}\left(\dot{x}^{2}+\dot{y}^{2}\right)^{1 / 2} d t=b E(P, Q),
$$

and the proof of the lemma is complete.

Let $r$ be a geodesic ray, $s$ the arc length on $r$ measured from the initial point, $P(s)$ the point of $r$ determined by $s$, and 0 the origin. The geodesic ray $r$ will be said to recede to infinity if $\lim _{s \rightarrow+\infty} E(0, P(s))=+\infty$. An unending geodesic $g$ will be said to recede to infinity if each of the geodesic rays into which $g$ is divided by any point recedes to infinity.

As a rather evident consequence of Lemma 2.1, we can state the following theorem.

Theorem 2.1. Every class $A$ geodesic ray and every class $A$ unending geodesic on $M(F)$ recedes to infinity.

We shall subsequently be concerned with the question of the extent to which geodesic segments, geodesic rays, or unending geodesics of class $A$ behave like euclidean line segments, half-lines or whole lines, respectively. To that end we introduce the following well known definitions.

If $U$ is a set of points in $\Theta$ and $P$ is a point of $\Theta$, we define the distance of the point $P$ from the set $U$, or $D(P, U)$, by

$$
D(P, U)=\underset{u \in U}{\text { g.l.b. }} D(P, u) .
$$

If $U$ and $V$ are point sets in $\Theta$ we define (cf. Hausdorff $[1$, p. 146]) the typedistance between these sets, or $D(U, V)$, by

$$
D(U, V)=\max (\underset{u \in U}{\text { l.u.b. }} D(u, V) ; \underset{v \in V}{\text { l.u.b. }} D(v, U)) .
$$

If either of the bounds on the right does not exist, $D(U, V)=+\infty$.

Two sets $U$ and $V$ will be said to be of the same type, or each will be said to be of the type of the other, if $D(U, V)$ is finite.

If there were a uniform upper bound $R$ of the type-distance between a class $A$ geodesic segment and the $E$-line (euclidean line) segment with the same end points, we could infer the existence of a large class of unending geodesics of class $A$. As has been stated, the hypotheses made up to this point do not imply the existence of any class $A$ unending geodesics, so that additional restrictions must be imposed to secure the existence of the constant $R$

3. Doubly-periodic Riemannian manifolds. Let $T_{1}$ and $T_{2}$ be translations of $\Theta$ into $\Theta$ such that the fixed lines of $T_{1}$ and $T_{2}$ are not identical. Let $G$ be the doubly-periodic group with $T_{1}$ and $T_{2}$ as generators.

The condition that $F(x, y)$ be invariant under the translations of $G$ will be 
denoted by (I). A two-dimensional Riemannian manifold $M(F)$ which satisfies (I) will be termed a doubly-periodic Riemannian manifold and denoted by $M(F, \mathrm{I})$.

The following theorem has been proved by Hedlund. (Cf. Hedlund [1, p. 731]. The groups of translations considered in Hedlund [1] were made up only of translations parallel to the axes, but the arguments used in the proofs of the theorems require no essential modifications in order to apply to the group $G$ of the present paper. The corresponding theorem of Hedlund [1] is stated in terms of $E$-distance, but Lemma 2.1 permits restatement in terms of the type-distance defined here.)

THEOREM 3.1. Corresponding to a given $M(F, \mathrm{I})$ there exists a finite constant $R$ such that the type-distance between any class $A$ geodesic segment on $M(F, \mathrm{I})$ and the E-line segment with the same end points cannot exceed $R$.

As a consequence of this theorem, the following can be deduced (cf. Hedlund $[1$, p. 732$])$.

TheOREM 3.2. Each unending geodesic of class $A$ on an $M(F, \mathrm{I})$ is of the type of some E-line. Conversely, corresponding to an arbitrary E-line L, there is at least one unending class $A$ geodesic of the type of $L$.

We will term a closed half-line of $\Theta$ a euclidean ray or $E$-ray.

THeOREM 3.3. Each geodesic ray $r$ of class $A$ on an $M(F, \mathrm{I})$ is of the type of a unique E-ray $l$ with the same initial point and the type-distance between $l$ and $r$ does not exceed the constant $R$ of Theorem 3.1. Conversely, corresponding to any E-ray $l$ there exists a class $A$ geodesic ray $r$ on $M(F, \mathrm{I})$ of the same type and with the same initial point as $l$. The type distance between $l$ and $r$ does not exceed the constant $R$.

Let $r$ be a class $A$ geodesic ray with initial point $P_{0}$ and let $s$ be the arc length on $r$ measured from $P_{0}$. Let $P_{n}$ be the point on $r$ such that the value of $s$ determining $P_{n}$ is $n$. According to Theorem 2.1, $r$ recedes to infinity and consequently as $n$ becomes infinite the $E$-distance $P_{0} P_{n}$ must become infinite. If the sequence $0<n_{1}<n_{2}<\cdots$ is properly chosen, the sequence of $E$-rays $l_{1}, l_{2}, \cdots$, where $l_{i}$ has the initial point $P_{0}$ and passes through $P_{n_{i}}$, will have a unique limiting $E$-ray $l$ with initial point $P_{0}$. According to Theorem 3.1, the type-distance between the segment $P_{0} P_{n_{i}}$ of $r$ and the $E$-line segment $P_{0} P_{n_{i}}$ does not exceed $R$. It follows that the type-distance between $l$ and $r$ does not exceed $R$. Thus the existence of at least one $E$-ray $l$ corresponding to $r$ and with the stated properties has been proved.

To show that $l$ is unique, suppose a second $E$-ray $l^{\prime}$ exists with initial point $P_{0}$ and such that $r$ is also of the type of $l^{\prime}$. Since $l$ and $l^{\prime}$ are both of the type of $r$, it would follow that $l$ and $l^{\prime}$ are of the same type and this is evidently not the case. 
Conversely, given an $E$-ray $l$, let $P_{0}, P_{1}, \cdots$ be an ordered sequence of points on $l$ such that $P_{0}$ is the initial point of $l$ and the $E$-distance $P_{0} P_{n}$ becomes infinite with $n$. If $g_{n}$ is a class $A$ geodesic segment joining $P_{0}$ and $P_{n}$, it follows from Theorem 3.1 that $g_{n}$ cannot recede a distance exceeding $R$ from the $E$-line segment $P_{0} P_{n}$. If $g_{n}$ is oriented so that $P_{0}$ is its initial point, let $e_{n}$ denote the element of $g_{n}$ at $P_{0}$. That is, $e_{n}$ is a triple of numbers $\left(x_{0}, y_{0}, \phi_{n}\right)$, where $\left(x_{0}, y_{0}\right)$ are the coordinates of $P_{0}$ and where $\phi_{n}$, with $0 \leqq \phi_{n} \leqq 2 \pi$, is the angular coordinate at $P_{0}$ determined by the direction of $g_{n}$ at $P_{0}$. The sequence $\phi_{1}, \phi_{2}, \ldots$ contains a subsequence converging to some value $\phi$ and we say that the corresponding subsequence of $e_{1}, e_{2}, \ldots$ converges to the element $e\left(x_{0}, y_{0}, \phi\right)$. The geodesic ray $r$ with initial element $e$ must be of class $A$, for it is the limit of class $A$ geodesic segments $g_{n}$. Since no point of $g_{n}$ can be at a distance exceeding $R$ from the $E$-line segment $P_{0} P_{n}$, it follows that no point of $r$ can be at a distance from $l$ exceeding $R$.

On the other hand, if $Q$ is any point of $l, Q$ is a point of $P_{0} P_{n}$ for $n$ greater than some properly chosen integer $N$. It follows from Theorem 3.1 that for $n>N, g_{n}$ has on it a point $Q_{n}$ such that $D\left(Q, Q_{n}\right) \leqq R$. Since $g_{n}$ is of class $A$, the length of the segment $P_{0} Q_{n}$ of $g_{n}$ is less than a constant independent of $n$. In fact

$$
D\left(P_{0}, Q_{n}\right) \leqq D\left(P_{0}, Q\right)+D\left(Q, Q_{n}\right) \leqq D\left(P_{0}, Q\right)+R .
$$

It follows that the sequence $Q_{1}, Q_{2}, \cdots$ must contain a subsequence which converges to a point $Q^{\prime}$ of $r$. But then $D\left(Q, Q^{\prime}\right) \leqq R$, and no point of $l$ can be at a distance exceeding $R$ from $r$. Thus $r$ and $l$ are of the same type with typedistance not exceeding $R$ and the proof of Theorem 3.3 is complete.

ThForem 3.4. Let $g_{1}$ and $g_{2}$ be class $A$ unending geodesics on an $M(F, \mathrm{I})$ such that $g_{1}$ and $g_{2}$ are not identical and intersect in a point $P$. Corresponding to $\epsilon>0$, there exists $a \delta>0$ such that, if $g_{1}^{\prime}$ and $g_{2}^{\prime}$ are those point sets of $g_{1}$ and $g_{2}$, respectively, which are at distance at least $\epsilon$ from $P$, the distance between any point of $g_{1}^{\prime}$ and any point of $g_{2}^{\prime}$ cannot be less than $\delta$.

The proof of this theorem is essentially the same as that of Theorem 6 of Morse [1]. If Theorem 3.4 were not true, there would exist an $\epsilon>0$, a sequence $\delta_{1}>\delta_{2}>\cdots$, with $\lim _{i \rightarrow+\infty} \delta_{i}=0$, and a sequence of point pairs $\left(M_{1}, N_{1}\right)$, $\left(M_{2}, N_{2}\right), \cdots, M_{i}$ on $g_{1}, N_{i}$ on $g_{2}$, with both $M_{i}$ and $N_{i}$ at distance at least $\epsilon$ from $P$, and such that $\lim _{i \rightarrow+\infty} D\left(M_{i}, N_{i}\right)=0$. By proper choice of subsequences we can assume that $P$ does not separate on $g_{1}$ any two points $M_{i}$ and $M_{j}$, nor any two points $N_{i}$ and $N_{j}$ on $g_{2}$. Let $Q$ be a point on $g_{1}$, distinct from $P$ and such that on $g_{1}, P$ separates $Q$ and any point $M_{i}$. Then the length of the broken geodesic $\hat{g}_{i}$ consisting of the segment $Q P$ of $g_{1}$ and the segment $P N_{i}$ of $g_{2}$ does not exceed the length of the geodesic segment $Q M_{i}$ of $g_{1}$ by more than $\delta_{i}$. But the broken geodesic $\hat{g}_{i}$ has a corner at $P$ and the points $Q$ and $N_{i}$ can be joined by a geodesic segment the length of which is less than 
the length of $\hat{g}_{i}$ by a fixed amount which is independent of $i$. Since $\lim _{i \rightarrow+\infty} \delta_{i}=0$, we infer that for $i$ sufficiently large the segment $Q M_{i}$ of $g_{1}$ cannot be the shortest geodesic segment joining $Q$ and $M_{i}$, contrary to the hypothesis that $g_{1}$ is of class $A$.

The proof of the theorem is complete.

A set of points $U$ of the plane $\Theta$ will be said to be periodic with period $(m, n)$, if $U$ is invariant under the translation $T_{1}^{m} T_{2}^{n}, m^{2}+n^{2} \neq 0$, of $G$.

The period $(m, n), m>0$, is a primitive period if $m$ and $n$ are relatively prime. The period $(0, n)$ is a primitive period if $n=1$. Corresponding to any period $(m, n)$, there exists a unique primitive period $(\mu, \nu)$ such that $m=k \mu$, $n=k \nu, k$ integral. We term $(\mu, \nu)$ the primitive period corresponding to $(m, n)$. It is easily shown that if an $E$-line has the period $(m, n)$, it has all the periods and only the periods $(i \mu, i \nu), i= \pm 1, \pm 2, \cdots$, where $(\mu, \nu)$ is the primitive period corresponding to $(m, n)$.

TheOREM 3.5. If $g$ is an unending class $A$ periodic geodesic on an $M(F, \mathrm{I})$ and $g$ is of the type of the periodic E-line $L$ with primitive period $(\mu, \nu)$, then $g$ has all the periods and only the periods $(j \mu, j \nu), j= \pm 1, \pm 2, \cdots$.

For suppose that $g$ has the period $(m, n)$. Then $g$ is invariant under the transformation $T_{1}^{m} T_{2}^{n}$. We show that $T_{1}^{m} T_{2}^{n}=T$ transforms $L$ into itself.

Since $L$ and $g$ are of the same type, their type-distance is less than a fixed constant $C$. The metric $D(P, Q)$ is invariant under $T$ and all its powers, so that the type-distance between $T^{k}(L)$ and $T^{k}(g), k$ any positive integer, is less than $C$. But $T^{k}(g)=g$, and if $T(L)$ were not $L$, the type-distance between $T^{k}(L)$ and $L$ would increase without limit as $k$ becomes infinite. It would follow that for $k$ sufficiently large, the type-distance between $T^{k}(g)=g$ and $T^{k}(L)$ would exceed $C$. But this type-distance is less than $C$ and we infer that $T(L)=L$. Since $L$ has only periods of the form $(j \mu, j \nu), j$ integral, it follows that $m=i \mu, n=i \nu, i$ a nonvanishing integer.

It remains to show that $g$ actually has $(\mu, \nu)$ among its periods. If we let $T_{1}^{\mu} T_{2}^{\nu}=\bar{T}$, it follows that $\bar{T}^{i}=T$. Suppose that $g$ is not invariant under $\bar{T}$. Then $\bar{T}(g)=g^{\prime}$ is periodic and not identical with $g$. Moreover

$$
T\left(g^{\prime}\right)=T(\bar{T}(g))=\bar{T}(T(g))=\bar{T}(g)=g^{\prime} .
$$

Thus $g$ and $g^{\prime}$ are both invariant under $T$. Hence $g$ and $g^{\prime}$, both of which are of class $A$, can have no points in common. The unending geodesic $g$ divides the plane $\Theta$, excluding $g$, into two open sets $\Theta_{1}$ and $\Theta_{2}$ in one of which $g^{\prime}$ lies. We can assume that $g^{\prime}$ lies in $\Theta_{2}$. The geodesic $g^{\prime}$ divides the plane $\Theta$ into two open sets $\Theta_{1}^{\prime}$ and $\Theta_{2}^{\prime}$, of which one, and we assume it to be $\Theta_{1}^{\prime}$, contains $g$, and hence $\Theta_{1}$. Then $\bar{T}\left(\Theta_{1}\right)=\Theta_{1}^{\prime}$ and $\bar{T}\left(\Theta_{1}\right)$ contains $\Theta_{1}$ and additional points. But $\bar{T}^{2}\left(\Theta_{1}\right)=\bar{T}\left(\Theta_{1}^{\prime}\right)$ contains $\Theta_{1}$ and additional points. In general, $\bar{T}^{k}\left(\Theta_{1}\right)$ contains $\Theta_{1}$ and additional points, if $k>0$. Since $\bar{T}^{i}(g)=g$, it follows that $\bar{T}^{i}\left(\Theta_{1}\right)=\Theta_{1}$ and we have a contradiction, if $i$ is positive. Similar arguments apply if $i$ is negative. 
The proof of the theorem is complete.

4. A class of closed orientable manifolds of genus one. Let $M(F, \mathrm{I})$ be a doubly-periodic Riemannian manifold. Then $F(x, y)$ is invariant under a group $G$ of translations. We note that the assumption that $F$ satisfies (2.2) is now implied by the assumption $F>0$. For $F$ is continuous and assumes all its values in a parallelogram. It follows that $F$ has a positive lower bound and a positive upper bound.

Since $F$ is invariant under the group $G$, the metric (2.1) is invariant under the transformations of $G$. If points of the $(x, y)$-plane which are congruent under a transformation of $G$ are considered identical, there is defined a closed orientable two-dimensional Riemannian manifold $M(F, G)$ of genus one. A geodesic on $M(F, G)$ is represented in $\Theta$ by an infinite set of congruent geodesics of $M(F)$.

If $(x, y)$ is a point of $\Theta$ and $0 \leqq \phi<2 \pi$, the triple of numbers $(x, y, \phi)$ determines a direction $\phi$ at the point $(x, y)$ of $\Theta$ if we assume that $\phi$ is measured in the positive sense from a direction parallel to the positive $x$-axis. We term the point $P(x, y)$ the point bearing the element $(x, y, \phi)$. Let $\varepsilon$ denote the set of elements $(x, y, \phi),(x, y)$ in $\Theta, 0 \leqq \phi<2 \pi$. We topologize the space $\varepsilon$ by considering it as the product of the plane $\Theta$ and the unit circle.

A transformation of $G$ transforms a point of $\varepsilon$ into a congruent point or element and we denote by $\Omega$ the space obtained by identifying congruent points (elements) of $\mathcal{E}$. The space $\Omega$ is the space of elements on $M(F, G)$ and is a three-dimensional torus. A point in $\Omega$ is represented in $\Theta$ by an infinite set of congruent elements.

The directed geodesics on $M(F, G)$ define a continuous flow $T_{s}$ in the space $\Omega$, where $s$ is the arc length along the geodesics (cf., e.g., Hedlund [3]). If we define measure in $\Omega$ by means of the integral

$$
\iiint F^{2}(x, y) d x d y d \phi,
$$

the transformation $T_{s}$ is measure-preserving for each real $s$. It is evident that $m \Omega<+\infty$.

A motion in $\Omega$ is any set $T_{s} p$, with $-\infty<s<+\infty$, and $p$ a point of $\Omega$. A motion in $\Omega$ is the set of elements on a directed geodesic on $M(F, G)$ and is represented in $\Theta$ by an infinite set of congruent directed unending geodesics of $M(F)$.

A motion in $\Omega$ defined by the set $T_{s} p$ is said to be stable in the sense of Poisson, if there exists an infinite sequence of values $\cdots<s_{-1}<s_{0}<s_{1}<s_{2}<\cdots$, with $\lim _{i \rightarrow \infty}\left|s_{i}\right|=+\infty$, such that $\lim _{|i| \rightarrow+\infty} T_{s_{i}}(p)=p$. Let $M$ be a motion which is stable in the sense of Poisson and let $g$ be a directed unending geodesic of $\Theta$ representing $M$. If $s$ denotes the directed arc length on $g$ measured from some fixed point $P_{0}$, there must exist an infinite sequence $\cdots<s_{-1}<s_{0}<s_{1}<\cdots$, with $\lim _{|i|++\infty}\left|s_{i}\right|=+\infty$, and a sequence of points 
$\cdots, Q_{-1}, Q_{0}, Q_{1}, \cdots$, each congruent to $P_{0}$, such that if $P_{i}$ denotes the point of $g$ determined by $s_{i}, \lim _{|n| \rightarrow+\infty} D\left(P_{n}, Q_{n}\right)=0$.

Since $m \Omega<+\infty$, it follows from the well known recurrence theorem of Poincaré (cf. Poincaré [1], Carathéodory [1], E. Hopf [1]) that almost all points of $\Omega$ are on motions which are stable in the sense of Poisson. We will make extensive use of this theorem.

5. Properties of the geodesics on doubly-periodic manifolds which satisfy the non-conjugacy hypothesis. We make the following definition.

(II) Non-CONJUgacy hyPOTHEsis. There is no pair of mutually conjugate points on any geodesic on $M(F)$.

Theorem 5.1. A necessary and sufficient condition that $M(F)$ fulfill the nonconjugacy hypothesis is that there be only one geodesic segment joining two given points of $M(F)$.

If there are no two mutually conjugate points on any geodesic $g$ on $M(F)$, the geodesic rays issuing from a point $P$ of $\Theta$, form a field in $\Theta$ in the sense that, if $Q$ is any point of $\Theta$ other than $P$, there is one and only one geodesic ray with initial point $P$ and passing through $Q$. It follows that there is just one geodesic segment joining two points of $M(F)$.

Conversely, if a geodesic $g$ on $M(F)$ has on it two mutually conjugate points, a segment $\sigma$ of $g$ containing these mutually conjugate points as interior points is not of class $A$ and the end points of $\sigma$ can be joined by a class $A$ geodesic segment which differs from $\sigma$. Thus, if the geodesic segment joining any two given points of $M(F)$ is unique, the non-conjugacy hypothesis is fulfilled.

Theorem 5.2. A necessary and sufficient condition that $M(F)$ fulfill the nonconjugacy hypothesis is that all geodesics on $M(F)$ be of class $A$.

The necessity follows at once from Theorem 5.1.

The sufficiency follows as in the proof of Theorem 5.1.

We will denote a manifold $M(F, \mathrm{I})$ which fulfills the non-conjugacy hypothesis by $M(F, \mathrm{I}, \mathrm{II})$.

It follows from Theorems 3.2 and 5.2 that each unending geodesic on an $M(F, \mathrm{I}, \mathrm{II})$ is of the type of an $E$-line. It is evident that two unending geodesics on $M(F, \mathrm{I}, \mathrm{II})$ which are of the types of non-parallel $E$-lines must cross and thus they intersect in just one point. The question arises as to whether two unending geodesics on $M(F$, I, II) which are of the types of parallel $E$-lines, or, what is equivalent, of the same type, can cross. As we shall see, the answer is in the negative.

Lemma 5.1. Two unending geodesics on an $M(F$, I, II) which are of the type of the same periodic E-line cannot intersect. 
For suppose that $g_{1}$ and $g_{2}$ are intersecting unending geodesics on $M(F, \mathrm{I}, \mathrm{II})$ and each is of the type of the periodic $E$-line $L$. Let $(\mu, \nu)$ be the primitive period of $L$. It follows from Theorem 5.2 that both $g_{1}$ and $g_{2}$ are of class $A$. We consider the various possibilities.

Case I. $g_{1}$ and $g_{2}$ are both periodic. It follows from Theorem 3.5 that both $g_{1}$ and $g_{2}$ have the period $(\mu, \nu)$. Since $g_{1}$ and $g_{2}$ intersect in a point $P$, it would follow that they intersect in the point $T_{1}^{\mu} T_{2}^{\nu}(P) \neq P$. Since $g_{1}$ aad $g_{2}$ are both of class $A$, they cannot intersect in two points and we infer that the hypothesis of Case I is impossible.

Case II. One of the pair $g_{1}, g_{2}$ is periodic, but not both are periodic. It is known (cf. Hedlund [1, Theorem XIII]) that a class $A$ unending geodesic $g$ which is of the type of a periodic $E$-line $L$ is either periodic or is asymptotic in both its senses to periodic class $A$ geodesics which are of the type of $L$. It is also known (cf. Hedlund [1, Theorem XV]) that a geodesic $g$ of class $A$ which is asymptotic to a periodic geodesic $b$ of class $A$ cannot cross any class $A$ periodic geodesic of the type of $b$. These two statements are incompatible with the hypothesis of Case II and we infer that Case II is not possible.

Case III. Neither $g_{1}$ nor $g_{2}$ is periodic. The point $P$ in which $g_{1}$ and $g_{2}$ intersect divides $g_{1}\left(g_{2}\right)$ into two geodesic rays $r_{1}$ and $s_{1}\left(r_{2}\right.$ and $\left.s_{2}\right)$, all four geodesic rays thus determined having the point $P$ as initial point and no two of the four intersecting in any point other than $P$. Since $g_{1}$ and $g_{2}$ are of the same type, the four geodesic rays can be divided into two pairs such that members of the same pair are of the same type. We assume that the notation has been so chosen that $r_{1}$ and $r_{2}$ are of the same type.

The rays $r_{1}$ and $r_{2}$ divide the points of the plane $\Theta$ which are not on these rays into two open connected sets $\sigma$ and $\cdot \lambda$ which are separated by the set consisting of the points of $r_{1}$ and $r_{2}$. (This is easily seen by projecting the plane stereographically onto the unit sphere. Then the two rays $r_{1}$ and $r_{2}$, together with the north pole of the sphere, form a Jordan curve on the sphere.) It follows from Theorem 3.3 that a half-strip $\Gamma$ (i.e., the set of points between two parallel lines and on one side of a line perpendicular to these two) can be so drawn that $r_{1}$ and $r_{2}$ lie in $\Gamma$. But then one of the sets $\sigma$ or $\lambda$ (and we assume that it is $\sigma$ ) lies entirely in $\Gamma$, and hence is of the type of $r_{1}$ (and $r_{2}$ ).

Let $g$ be a geodesic which passes through $P$ and enters the set $\sigma$. Then $P$ divides $g$ into two rays of which one, which we denote by $r$, lies entirely in $\sigma$. It follows from Theorem XIII of Hedlund [1], that either $g$ is periodic and of the type of $L$ or $r$ is asymptotic to a periodic (class $A$ ) geodesic $b$ which is of the type of $L$. The first possibility is ruled out by applying Case II to the pair $g$ and $g_{1}$. If the second possibility holds, it follows from Theorem 3.4 that $b$ has on it points in $\sigma$. But $b$ must leave $\sigma$ and hence cross either $r_{1}$ or $r_{2}$. Again we have Case II by use of $g_{1}$ and $b$ if $r_{1}$ and $b$ cross, or by use of $g_{2}$ and $b$ if $r_{2}$ and $b$ cross.

The proof of the theorem is complete. 
TheOREM 5.3. Any unending geodesic $g$ on an $M(F, \mathrm{I}, \mathrm{II})$ which is of the type of a periodic E-line is periodic.

For let $g$ be an unending geodesic on $M(F$, I, II $)$ and of the type of the periodic $E$-line $L$. Then $g$ is of class $A$, and if $g$ is not periodic, we have seen (Hedlund [1, Theorem XIII]) that $g$ is asymptotic in both its senses to periodic class $A$ geodesics $b$ and $c$ of the type of $L$. But a geodesic of class $A$ cannot be asymptotic in both its senses to the same periodic geodesic (cf. Hedlund [1, Theorem XVII]) so that $b$ and $c$ are distinct. Since $b$ and $c$ are of class $A$ and periodic, they can have no points in common. There can be no class $A$ periodic geodesic lying between $b$ and $c$, for such a geodesic would cross $g$, which is impossible. But then there must exist two different class $A$ geodesics which lie between $b$ and $c$ and which must intersect (cf. Hedlund $[1$, Theorem XVI]). In view of Lemma 5.1 this cannot be the case. We infer that $g$ must be periodic and the proof of the theorem is complete.

TheOREM 5.4. An unending geodesic on an $M(F$, I, II) which passes through two congruent points is periodic.

For let $P$ and $Q$ be a pair of congruent points of $\Theta$ and let $g$ be an unending geodesic passing through $P$ and $Q$. Let $l$ be the $E$-ray with initial point $P$ and passing through $Q$. Then $l$ is part of a periodic $E$-line $L$ with primitive period $(\mu, \nu)$. According to Theorem 3.3 there exists a class $A$ geodesic ray $r$ of the type of $l$ and with initial point $P$. Since all geodesics on $M(F, \mathrm{I}, \mathrm{II})$ are of class $A$, the unending geodesic $g^{*}$, of which $r$ is a part, is of the type of an $E$-line and must be of the type of $L$. From Theorem 5.3 we infer that $g^{*}$ is periodic and from Theorem 3.5 that $g^{*}$ has the period $(\mu, \nu)$. But then $g^{*}$ must pass through $Q$ and thus is identical with $g$. It follows that $g$ is periodic.

THEOREM 5.5. Two unending geodesics on an $M(F, \mathrm{I}, \mathrm{II})$ which are of the same type cannot intersect.

For suppose $g_{1}$ and $g_{2}$ are unending geodesics on $M(F, \mathrm{I}, \mathrm{II})$ which are of the same type and intersect in the point $P$. It follows from Theorem 5.1 that $g_{1}$ and $g_{2}$ can intersect in no other point than $P$. As in the proof of Lemma 5.1, the point $P$ divides $g_{1}\left(g_{2}\right)$ into two geodesic rays $r_{1}$ and $s_{1}\left(r_{2}\right.$ and $\left.s_{2}\right)$, all four geodesic rays thus determined having the point $P$ as initial point and no two intersecting in any point other than $P$. We can assume the notation has been so chosen that $r_{1}$ and $r_{2}\left(s_{1}\right.$ and $\left.s_{2}\right)$ are of the same type and neither $r_{1}$ nor $r_{2}$ is of the type $s_{1}$ or $s_{2}$.

The rays $r_{1}$ and $r_{2}$ divide the points of $\Theta$ which are not on these rays into two open connected sets of which one, which we denote by $\sigma$, is of the type of $r_{1}$ (or $r_{2}$ ). Similarly the rays $s_{1}$ and $s_{2}$ divide the plane $\Theta$ into two connected open sets of which one, which we denote by $\lambda$, is of the type of $s_{1}$ (or $s_{2}$ ). The set $\sigma$ can contain no points of $s_{1}$ (or $s_{2}$ ), for if this were the case, $\sigma$ would contain all points of $s_{1}$ (or $s_{2}$ ) except $P$, and could not be of the type of $r_{1}$ (or $r_{2}$ ). 
Similarly, $\lambda$ contains no points of $r_{1}$ or $r_{2}$. It follows that either $\lambda$ lies entirely in $\sigma$ or $\lambda$ contains no point of $\sigma$. If the first were true, $\lambda$ would be of the type of $\sigma$, and thus of the type of $r_{1}$ (or $r_{2}$ ). Since this is not the case, we infer that $\lambda$ contains no point of $\sigma$. Similarly $\sigma$ contains no point of $\lambda$.

Any geodesic segment $g^{*}$ which joins a point $T$ in $\sigma$ and a point $U$ in $\lambda$ must cross one of the rays $s_{1}$ or $s_{2}$, and one of the rays $r_{1}$ or $r_{2}$. Since $g^{*}$ can cross $g_{1}$, which is made up of $r_{1}$ and $s_{1}$, in at most one point and, similarly, $g_{2}$ in at most one point, $g^{*}$ must cross both $g_{1}$ and $g_{2}$. If $g$ is the unending geodesic of which $g^{*}$ is a segment, since $g$ is of class $A, g$ cannot leave the set $\sigma+\lambda$ except for a finite segment of $g^{*}$ and consequently $g$ must be of the type of $g_{1}$ (or $g_{2}$ ).

Now consider the set $G$ of oriented geodesics each of which has a finite segment with the initial point in $\lambda$ and the terminal point in $\sigma$. All oriented geodesics on the closed manifold $M(F, G)$ which are represented by geodesics in the set $G$ form a set $\bar{G}$ of geodesics on $M(F, G)$. The set of elements on the geodesics of $\bar{G}$ is evidently an open set $\alpha$ in $\Omega$ and since $\alpha$ is an open set it is measurable and of positive measure. According to the Poincaré recurrence theorem the set $\bar{G}$ must contain a motion (geodesic) $\bar{g}$ which is stable in the sense of Poisson. Let $g$ be an unending geodesic in the set $G$ and representing $\bar{g}$. If $s$ denotes the directed arc length on $g$ measured from some point $P_{0}$, there must exist an infinite sequence $\cdots<s_{-1}<s_{0}<s_{1}<s_{2}<\cdots$ with $\lim _{|n| \rightarrow \infty}\left|s_{n}\right|=+\infty$, and a sequence of points $\cdots, Q_{-1}, Q_{0}, Q_{1}, \cdots$, each congruent to $P_{0}$, and such that, if $P_{i}$ denotes the point of $g$ determined by $s_{i}, \lim _{|n| \rightarrow+\infty} D\left(P_{n}, Q_{n}\right)=0$. By application of Theorem 3.4 to each of the pairs $g, g_{1}$ and $g$, $g_{2}$, we infer that infinitely many of the points $\cdots, Q_{-1}, Q_{0}, Q_{1}, \cdots$ are in each of the sets $\sigma$ and $\lambda$. In particular, suppose that $Q_{\mu}$ is in $\lambda$ and $Q_{\nu}$ is in $\sigma$. Then according to Theorem 5.4 the geodesic $g_{P}$ determined by the congruent points $Q_{\mu}$ and $Q_{\nu}$ is periodic. The periodic geodesic $g_{P}$ is of the type of $g_{1}$ (or $g_{2}$ ), and it follows that $g_{1}$ and $g_{2}$ are of the type of the same periodic $E$-line $L$. But according to Lemma 5.1, it is impossible for $g_{1}$ and $g_{2}$ to intersect, as we have assumed.

The proof of Theorem 5.5 is complete.

THEOREM 5.6. If $g$ is an unending geodesic on an $M(F, \mathrm{I}, \mathrm{II})$ and $P$ is a point not on $g$, there exists one and only one unending geodesic on $M(F, \mathrm{I}, \mathrm{II})$ which passes through $P$ and does not intersect $g$.

It follows from Theorem 5.2 that $g$ is of class $A$ and thus according to Theorem 3.2, $g$ is of the type of an $E$-line $L$. If $l$ denotes an $E$-ray with initial point $P$ and of the type of one of the rays into which $L$ is divided by a point $Q$, it follows from Theorem 3.3 that there exists a geodesic ray $r$ with initial point $P$ and of the type of $l$. If $g^{*}$ is the unending geodesic of which $r$ is a subset, $g^{*}$ is of class $A$, of the type of an $E$-line, and hence of the type of $L$. Since $P$ is not on $g, g^{*}$ is not identical with $g$, and since both $g$ and $g^{*}$ are of the type of $L$, they are of the same type. We infer from Theorem 5.5 that $g$ and $g^{*}$ cannot intersect. 
If there were two unending geodesics passing through $P$ and each of the type of $g$, we would have two intersecting geodesics of the same type, contradictory to Theorem 5.5.

The proof of the theorem is complete.

The preceding theorem can be stated as follows.

THEOREM 5.7. The geodesics on an $M(F, \mathrm{I}, \mathrm{II})$ can be grouped into $\aleph$ families, the members of any one family forming a field in $\Theta$, and any two members from different families intersecting in just one point.

For let $L$ be an $E$-line. There exists an unending geodesic $g$ of the type of $L$. If $P$ is any other point of $\Theta$ not on $g$, it follows from Theorem 5.6 that there is just one unending geodesic $g_{P}$ passing through $P$ and not intersecting $g$. The geodesic $g_{P}$ is of the type of $g$ and hence of the type of $L$. Thus the set $G_{L}$ of unending geodesics of the type of $L$ cover the plane $\Theta$. But according to Theorem 5.5, no two members of the set $G_{1}$ can intersect and thus the set $G_{L}$ forms a field in $\Theta$. No two of the $E$-lines passing through the origin are of the same type, and the existence of $\boldsymbol{N}$ families is evident. The last statement of the theorem follows from the fact that unending geodesics which are not of the same type must intersect, and since all geodesics are of class $A$ there can be at most one point of intersection.

6. Non-conjugacy and closed orientable surfaces of genus one. Let $M$ be a two-dimensional Riemannian manifold of class $C^{3}$ of the topological type of a torus. That is, $M$ is a two-dimensional topological manifold which is homeomorphic to a torus; with each point $p$ of $M$ there is associated a neighborhood $N_{p}$ which is mapped by a homeomorphism in to the interior of the unit circle, $x^{2}+y^{2}<1$, such that the transformation $T$ defined by overlapping neighborhoods is of class $C^{4}$ with nonvanishing Jacobian; a metric is assigned to each neighborhood $N_{p}$ by assigning a quadratic differential form $d s^{2}=f_{p}(x, y)\left(d x^{2}+d y^{2}\right)$ to the unit circle, $f_{p}(x, y)$ being of class $C^{3}$ and positive, and such that the transformation $T$ defined by overlapping neighborhoods transforms one of the corresponding quadratic forms into the other.

The manifold $M$ is a Riemann surface (cf. Koebe [1]) and its universal covering surface can be mapped conformally into the plane $\Theta$, thereby defining a metric of the form (2.1) which satisfies (2.2) and (I). If we denote this latter manifold by $M(F, \mathrm{I}), M$ is obtained from $M(F, \mathrm{I})$ by identification of congruent points. Thus $M$ is the manifold $M(F, G)$.

If there are no two mutually conjugate points on any geodesic on $M$, the corresponding Riemannian manifold defined in $\Theta$ is an $M(F$, I, II $)$. It follows that the theorems of $\S 5$ are applicable to the surface $M$. We state the principal results in the following theorem.

Theorem 6.1. Let $M$ be an orientable two-dimensional Riemannian manifold of genus one and of class $C^{3}$, such that no geodesic on $M$ has on it two mutually conjugate points. Any unending geodesic on $M$ is either a simple closed 
curve or the topological image of a line. The unending geodesics on $M$ can be grouped into $\boldsymbol{N}$ families such that the members of any one family cover $M$, no two members of the same family intersect and any two members of different families do intersect.

The statements of this theorem follow readily from Theorems 5.4 and 5.7.

7. Non-conjugacy and closed non-orientable surfaces of genus two. Let $M$ be a two-dimensional Riemannian manifold of the topological type of the Klein bottle (cf. Seifert-Threlfall [1, chap. 6]), or, as we shall term it, a closed non-orientable manifold of genus 2. (A Klein bottle is a closed non-orientable two-dimensional manifold with Euler characteristic 0.) The universal covering manifold of $M$ can be mapped conformally onto $\Theta$ (cf. Koebe [1]) and we obtain a quadratic form $(2.1)$ with $F(x, y)$ of class $C^{3}$ and invariant under a group $G$ which, in this case, contains inversely conformal transformations of $\Theta$ into itself, as well as translations. The manifold $M$ is obtained by identification of points which are congruent under $G$. The subset of transformations of $G$ which are translations form a subgroup $G^{*}$ which is generated by two linearly independent translations. The manifold $M$ can be considered as obtained by identifying points congruent under $G^{*}$, thus obtaining a closed orientable manifold $M^{*}$ of genus one, and then identifying the points of $M^{*}$ in pairs under an inversely conformal transformation. The statements of the following theorem are then obtained with the aid of Theorems 5.4 and 6.1.

TheOREM 7.1. Let $M$ be a non-orientable two-dimensional Riemannian manifold of genus two, of class $C^{3}$, and such that no geodesic on $M$ has on it two mutually conjugate points. The unending geodesics on $M$ can be grouped into $\mathbf{N}$ families, of which $\boldsymbol{\aleph}_{0}$ are made up of periodic geodesics, such that at each point of $M$ there are in general two directions (in special cases one direction) which determine members of the same family.

8. The non-focality hypothesis. Let $g$ be an unending geodesic on a doubly-periodic manifold $M(F, \mathrm{I})$. Corresponding to an arbitrary geodesic $g_{1}$ orthogonal to $g$ at a point of $g$ the first focal point $Q$ of $g$ on $g_{1}$, taking $g_{1}$ in either sense, is well defined, or fails to exist (cf. Bolza [1, §39]). Each such point $Q$ will be termed a focal point of $g$.

(III) The NON-FoCALITy hypothesis. The Riemannian manifold $M(F$, I, II) will be said to satisfy the non-focality hypothesis if there exists a periodic L-line with the following property. Corresponding to each unending geodesic $g$ of the type of $L$ there are no focal points of $g$ on at least one side of $g$.

We denote a manifold $M(F, \mathrm{I}, \mathrm{II})$ which satisfies the non-focality hypothesis by $M(F$, I, II, III).

Lemma 8.1. Let $M(F$, I, II, III) satisfy the non-focality hypothesis with respect to the periodic E-line $L$. Then if $g$ is any unending geodesic on $M(F, \mathrm{I}, \mathrm{II}, \mathrm{III})$ 
of the type of $L$ and $P$ is any point of $\Theta$, there exists a unique geodesic passing through $P$ and orthogonal to $g$.

If $P$ is on $g$ the statement of the lemma is obvious.

If $P$ is not on $g$, the distance $D(P, Q), Q$ any point on $g$, has a minimum and assumes this minimum for some point $T$ of $g$. The geodesic $g^{*}$ passing through $P$ and $T$ is orthogonal to $g$. Thus the existence of at least one geodesic $g^{*}$ satisfying the desired condition is proved.

Since the non-conjugacy hypothesis is fulfilled, $g$ is of class $A, g$ is simple, and thus $g$ divides the points of $\Theta$ not on $g$ into two open sets $\Theta_{1}$ and $\Theta_{2}$ which are separated by $g$. The non-focality hypothesis implies that in at least one of the sets $\Theta_{1}, \Theta_{2}$, and we can assume it to be $\Theta_{1}$, there is no point $P$ with two geodesics passing through $P$ and orthogonal to $g$.

Suppose $g_{1}$ and $g_{2}$ are both orthogonal to $g$ at $P_{1}$ and $P_{2}$, respectively, and intersect in a point $Q$ which is necessarily in $\Theta_{2}$. Since $L$ is periodic and $g$ is of the type of $L$, it follows from Theorem 5.3 that $g$ is periodic. Accordingly there exists a geodesic $g_{1}^{*}$ which is congruent to $g_{1}$ and intersects $g$ orthogonally at a point $P_{1}^{*}$ such that $P_{2}$ lies on the open segment $P_{1} P_{1}^{*}$ of $g$. The subset of $g_{1}\left(g_{1}^{*}\right)$ in $\Theta_{1}$ is a ray which we denote by $r_{1}\left(r_{1}^{*}\right)$. Let the subset of $\Theta_{1}$ bounded by $P_{1} P_{1}^{*}, r_{1}$ and $r_{1}^{*}$ be denoted by $\alpha$. The geodesic $g_{2}$ enters $\alpha$ at $P_{2}$, $g_{2}$ can have no other point in common with $g$ other than $P_{2}$, and $g_{2}$ cannot intersect $r_{1}$ or $r_{1}^{*}$, for this would imply the existence of different geodesics orthogonal to $g$ and meeting in $\Theta_{1}$. But then one of the rays into which $P_{2}$ divides $g_{2}$ is of the type of $r_{1}$ (or $r_{1}^{*}$ ). It follows that $g_{2}$ is of the type of $g_{1}$ and these geodesics cannot intersect in $Q$ as was assumed.

The proof of the lemma is complete.

THEOREM 8.1. The Gaussian curvature of an $M(F$, I, II, III) vanishes identically.

Let $L$ be the periodic $E$-line with respect to which the non-focality hypothesis is fulfilled. All the geodesics on an $M(F$, I, II, III) which are of the type of $L$ are periodic (Theorem 5.3) and no two intersect (Theorem 5.5). If we denote this family by $\mathcal{F}$, we have seen (cf. Theorem 5.7 and proof) that the geodesics of $\mathcal{F}$ form a field in $\Theta$.

Let $g$ be any geodesic in the family $\mathcal{F}$. According to Lemma 8.1, the geodesics orthogonal to $g$ also form a field $\mathcal{F}^{*}(g)$ in $\Theta$. Since two class $A$ geodesics which are of different types intersect, it follows that the geodesics in $\mathcal{F}^{*}(g)$ are of the same type and include all the geodesics of this type.

Let $g_{1}$ and $g_{2}$ be two geodesics in the family $\mathcal{F}$. If $P_{1}$ is any point of $g_{1}$ and $P_{2}$ is any point of $g_{2}, D\left(P_{1}, P_{2}\right)$ is defined and varies continuously with $P_{1}$ and $P_{2}$. Since $g_{1}$ and $g_{2}$ are periodic with the same period, $D\left(P_{1}, P_{2}\right)$ assumes all its values, if $P_{1}$ is restricted to a properly chosen finite segment $\omega$ of $g_{1}$. It follows that $D\left(P_{1}, P_{2}\right)$ assumes its minimum for some pair $Q_{1}, Q_{2}, Q_{1}$ in $\omega$, $Q_{2}$ on $g_{2}$, and the geodesic segment $\rho$ joining $Q_{1}$ and $Q_{2}$ is orthogonal to both 
$g_{1}$ and $g_{2}$. The unending geodesic $g^{*}$ of which $\rho$ is a segment belongs to both the families $\mathcal{F}^{*}\left(g_{1}\right)$ and $\mathcal{F}^{*}\left(g_{2}\right)$ and hence these families are identical. Thus the family $\mathcal{F}^{*}(g)$ is uniquely determined by $L$, and we denote this family by $\mathcal{F}^{*}$.

Each of the families $\mathcal{F}$ and $\mathcal{F}^{*}$ forms a field in $\Theta$ and each member of $\mathcal{F}$ intersects each member of $\mathcal{F}^{*}$ orthogonally in a single point. It is well known that this implies that the Gaussian curvature $K(x, y)$ vanishes identically. If this were not the case, there would exist a point $(\bar{x}, \bar{y})$ such that $K(\bar{x}, \bar{y}) \neq 0$. The function $K(x, y)$ is continuous and consequently there would exist a circle $\nu$ with center $(\bar{x}, \bar{y})$ such that either $K(x, y)>0$ or $K(x, y)<0$ in $\nu$. But by proper choice of two members of the field $\mathcal{F}$ and two members of the field $\mathcal{F}^{*}$ we obtain a geodesic quadrilateral $q$ with angles all right angles and lying in $\nu$. The Gauss-Bonnet formula implies

$$
\iint_{q} K F^{2} d x d y=0
$$

and we have a contradiction. Thus $K(x, y) \equiv 0$ and the proof of Theorem 6.1 is complete.

If $M$ is a closed two-dimensional Riemannian manifold of class $C^{3}$, corresponding to any point $P$ on any geodesic $g$ on $M$ there exists a set of focal points determined by $P$ and $g$. We term the totality of focal points corresponding to all the points of $g$ the focal points corresponding to $g$. If there are no focal points corresponding to any geodesic on $M$, there are no two mutually conjugate points on any geodesic on $M$. If, in addition, $M$ is of the topological type of a torus or a non-orientable surface of genus two, the universal covering surface of $M$ can be mapped onto the plane $\Theta$ and is an $M(F$, I, II, III). If we term a Riemannian manifold for which the Gaussian curvature vanishes identically flat, Theorem 8.1 implies the sufficiency in the following theorem. The necessity is obvious.

Theorem 8.2. A two-dimensional Riemannian manifold $M$ of class $C^{3}$ which is either orientable of genus one or non-orientable of genus two is flat, if and only if there are no focal points corresponding to any geodesic on $M$.

\section{Part II. Manifolds OF HYPERBolic TYPE}

9. A class of simply-connected Riemannian manifolds of hyperbolic type. Let $U$ be the unit circle $u^{2}+v^{2}=1$ and let $\Psi$ be its interior. To $\Psi$ we assign the metric

$$
d s^{2}=\frac{4 f^{2}(u, v)\left(d u^{2}+d v^{2}\right)}{\left(1-u^{2}-v^{2}\right)^{2}}
$$

where $f(u, v)$ is of class $C^{3}$ in $\Psi$ and there exist positive constants $a$ and $b$ such that 


$$
a \leqq f(u, v) \leqq b,
$$$$
u^{2}+v^{2}<1
$$

We denote this two-dimensional Riemannian manifold by $M(f)$ and term it a Riemannian manifold of hyperbolic type.

The metric (9.1) assigns a length to any rectifiable curve $\gamma$ on $M(f)$ and we denote this length by $L(\gamma)$. The geodesics corresponding to (9.1) are of class $C^{2}$ in terms of arc length as parameter and in terms of initial conditions. There is a unique geodesic passing through a point of $\Psi$ in a given direction.

In the special case when $f(u, v) \equiv 1,(9.1)$ reduces to the well known Poincare metric which defines a hyperbolic geometry in $\Psi$. In this case the geodesics are arcs of circles orthogonal to $U$ and will be termed hyperbolic lines or H-lines. The hyperbolic length or $H$-length of a curve in $\Psi$ is the length of the curve under the assumption $f \equiv 1$. Given two points $P$ and $Q$ of $\Psi$, there is a unique $H$-line segment joining these points and the $H$-length of this segment is the $H$-distance $H(P, Q)$ between $P$ and $Q$.

Again we term a geodesic segment $g$ joining $P$ and $Q$ of class $A$ if $g$ affords an absolute minimum of length relative to all rectifiable curves on $M(f)$ joining $P$ and $Q$. The manifold $M(f)$ is complete in the sense of Hopf and Rinow, and corresponding to a given pair of points $P$ and $Q$ of $\Psi$ there exists a class $A$ geodesic segment joining $P$ and $Q$. We term the length of a class $A$ geodesic segment joining $P$ and $Q$ the distance between $P$ and $Q$ and denote it by $D(P, Q)$. This metric has the usual properties.

A geodesic ray or an unending geodesic is of class $A$, if every finite segment of the ray or unending geodesic, respectively, is of class $A$. Geodesic segments geodesic rays, or unending geodesics of class $A$ are evidently simple curves.

Lemma 9.1. If $P$ and $Q$ are arbitrary points of $M(f)$,

$$
a H(P, Q) \leqq D(P, Q) \leqq b H(P, Q) .
$$

For let $\gamma$ be a class $A$ geodesic segment joining $P$ and $Q$. We then have

$$
\begin{aligned}
D(P, Q)=L(\gamma) & =\int_{\gamma} \frac{2 f\left(\dot{u}^{2}+\dot{v}^{2}\right)^{1 / 2}}{1-u^{2}-v^{2}} d t \geqq a \int_{\gamma} \frac{2\left(\dot{u}^{2}+\dot{v}^{2}\right)^{1 / 2}}{1-u^{2}-v^{2}} d t \\
& =a H(\gamma) \geqq a H(P, Q) .
\end{aligned}
$$

If we let $h$ denote the $H$-line segment joining $P$ and $Q$, we have

$$
\begin{aligned}
D(P, Q)=L(\gamma) \leqq L(h) & =\int_{h} \frac{2 f\left(\dot{u}^{2}+\dot{v}^{2}\right)^{1 / 2}}{1-u^{2}-\dot{v}^{2}} d t \\
& \leqq b \int_{h} \frac{2\left(\dot{u}^{2}+\dot{v}^{2}\right)^{1 / 2}}{1-u^{2}-v^{2}} d t=b H(P, Q) .
\end{aligned}
$$

The proof of the lemma is complete.

Let $X$ and $Y$ be point sets of $\Psi$. If $P$ is any point of $\Psi$ we define the distance from the point $P$ to the set $X$, or $D(P, X)$, by 


$$
D(P, X)=\underset{x \in X}{\text { g.l.b. }} D(P, x) .
$$

We define the type-distance between the sets $X$ and $Y$, or $\mathcal{D}(X, Y)$, by

$$
\mathcal{D}(X, Y)=\max (\underset{x \in X}{\text { l.u.b. }} D(x, Y) ; \underset{y \in Y}{\text { l.u.b. }} D(y, X)) .
$$

Two sets will be said to be of the same type if their type-distance is finite.

THEOREM 9.1. There exists a finite constant $R$, determined by $f(u, v)$ such that the type-distance between any class $A$ geodesic segment of $M(f)$ and the $H$-line segment with the same end points never exceeds $R$.

This theorem is essentially proved in Morse [1]. The proof follows from Lemma 9.1 of the present paper and Lemmas 4, 5, 6, 7, 8 of Morse [1].

We can state the following theorems (cf. Morse [2, p. 54]).

THEOREM 9.2. Every class $A$ unending geodesic on an $M(f)$ is of the type of some H-line. Conversely, corresponding to an arbitrary $H$-line $h$, there exists at least one unending class $A$ geodesic on $M(f)$ of the type of $h$. The type-distance between an unending class $A$ geodesic and an $H$-line of the same type cannot exceed the constant $R$ of Theorem 9.1.

Theorem 9.3. On an $M(f)$ a class $A$ geodesic ray having $P$ as initial point is of the type of a uni $i_{i}$ ue $H$-ray having $P$ as initial point. Conversely, corresponding to an $H$-ray with initial point $P$, there exists a class $A$ geodesic ray on $M(f)$ with initial point $P$ and of the type of the $H$-ray. The type-distance between two geodesic rays of class $A$ of the same type and with the same initial point, or between a geodesic ray of class $A$ and an $H$-ray of the same type and with the same initial point cannot exceed $R$.

An $H$-line determines and is determined by its two points at infinity on $U$. It follows from Theorem 9.2 that an unending geodesic $g$ of class $A$ on an $M(f)$ has among its limit points (in the euclidean sense) two and only two points of $U$. We term these the points at infinity of $g$.

10. A class of two-dimensional Riemannian manifolds of hyperbolic type. A one-to-one analytic transformation of $\Psi$ into itself which leaves the metric

$$
d s^{2}=\frac{4\left(d u^{2}+d v^{2}\right)}{\left(1-u^{2}-v^{2}\right)^{2}}
$$

invariant is conformal and hence is either a linear fractional transformation of the form (cf. Ford [1, chap. 1])

$$
w=\frac{a z+\bar{c}}{c z+\bar{a}}
$$$$
a \bar{a}-c \bar{c}=1,
$$

or clse is the product of such a transformation and an inversion in a circle 
orthogonal to $U$. Let $G$ be a properly discontinuous group of these transformations. That is, $G$ fulfills the condition that the set of points congruent under $G$ to any point $P$ of $\Psi$ does not have $P$ as limit point. Groups such as $G$ are said to be of the first or second kind according as all or not all points of $U$ are limit points of fixed points of transformations of the group.

If $f(u, v)$ of $(9.1)$ is invariant under the transformations of $G$, the metric (9.1) is also invariant under these same transformations, and if congruent points are identified there is defined a manifold $M(f, G)$ with properties depending on the properties of $f$ and $G$. A geodesic on $M(f, G)$ is represented in $\Psi$ by a finite or denumerable set of congruent geodesics of $M(f)$.

Let $E$ denote the set of elements $(u, v, \phi)$, where $u^{2}+v^{2}<1$ and $0 \leqq \phi<2 \pi$. The point $(u, v, \phi)$ of $E$ determines a point $P(u, v)$ of $\Psi$ and a direction $\phi$ at $P$, where we assume that $\phi$ is measured from a direction parallel to the positive $u$-axis. The point $P(u, v)$ is the point bearing $e$. To topologize the set $E$ we consider $E$ as the topological product of $\Psi$ and a circle with central angle $\phi$.

A transformation of $G$ transforms a point of $E$ into a congruent point or element and we denote by $\Omega$ the space obtained by identifying congruent points of $E$. The space $\Omega$ is the space of elements on $M(f, G)$. A point of $\Omega$ is represented in $\Psi$ by a finite or infinite set of congruent elements.

We define measure in $\Omega$ by means of the integral

$$
\iiint \frac{4 f^{2}(u, v)}{\left(1-u^{2}-v^{2}\right)^{2}} d u d v d \phi
$$

It is well known that this measure is invariant under the flow $T_{\mathrm{s}}$ defined in $\Omega$ by the directed geodesics on $M(f, G)$ with $s$ the arc length on the geodesics.

Let $p$ be a point of $\Omega$. The set $T_{s} p,-\infty<s<+\infty$, will be termed the motion determined by $p$. A motion is the set of elements of $\Omega$ on a directed geodesic on $M(f, G)$. The motion determined by $p$ is stable in the sense of Poisson if there exists an infinite sequence $\cdots<s_{-1}<s_{0}<s_{1}<s_{2}<\cdots$ with $\lim _{|n| \rightarrow+\infty}\left|s_{n}\right|=+\infty$, such that $\lim _{|n| \rightarrow+\infty} T_{s_{n}} p=p$.

Definition. The manifold $M(f, G)$ will be said to be Poisson stable provided almost all points of $\Omega$ are on motions which are stable in the sense of Poisson. (Compare E. Hopf [2, p. 271].)

The class of manifolds $-M(f, G)$ which are Poisson stable includes all those for which $\Omega$ is of finite measure, and hence those manifolds $M(f, G)$ which are of finite area. In particular, if $G$ is a Fuchsian group of the first kind with a finite set of generators, $M(f, G)$ is Poisson stable.

If $f$ is invariant under the group $G$ and $M(f, G)$ is Poisson stable, we say that $M(f)$ is Poisson stable and denote it by $M(f, \mathrm{I})$.

11. The non-conjugacy hypothesis. We make the following definition.

(II) The Non-conjugacy hypothesis. There is no pair of mutually conjugate points on any geodesic on $M(f)$. 
The proofs of the following two theorems are similar to those of Theorems 5.1 and 5.2.

THEOREM 11.1. A necessary and sufficient condition that $M(f)$ fulfill the nonconjugacy hypothesis is that there be only one geodesic segment joining two given points of $M(f)$.

THEOREM 11.2. A necessary and sufficient condition that $M(f)$ fulfill the nonconjugacy hypothesis is that all geodesics on $M(f)$ be of class $A$.

If all the geodesics on $M(f)$ are of class $A$, it follows from Theorem 9.2 that each unending geodesic is of the type of an $H$-line, but we cannot conclude that there is only one geodesic of the type of a given $H$-line. Under the same condition, each geodesic ray on $M(f)$ is of class $A$ and it follows from Theorem 9.3 that a geodesic ray issuing from a point $P$ of $\Psi$ is of the type of an $H$-ray issuing from $P$. It is conceivable however that there might be an infinite number of geodesic rays issuing from $P$ and of the type of the same $H$-ray.

We denote a manifold $M(f, \mathrm{I})$ which satisfies the non-conjugacy hypothesis by $M(f, \mathrm{I}, \mathrm{II})$.

12. Properties of the geodesics on an $M(f, \mathrm{I}, \mathrm{II})$. We first prove the following lemma.

Lemma 12.1. Let $P$ be a point of $\Psi$ and let $A$, on $U$, be a fixed point of $a$ hyperbolic transformation of $G$. If all the geodesics on $M(f)$ are of class $A$, there is but one geodesic ray. from $P$ tn $A$.

For suppose that there are two geodesic rays $r_{1}$ and $r_{2}$ from $P$ to $A$. It follows from a theorem of Morse (cf. Morse [1, Theorem 10]) that each of the geodesic rays issuing from $P$ and with $A$ as point at infinity is either periodic or asymptotic to a periodic geodesic and all such periodic geodesics are of the same type. No two of the geodesic rays from $P$ to $A$ can be asymptotic to the same periodic geodesic (cf. Morse [1, Theorem 6]). Let $r_{3}$ be a geodesic ray issuing from $P$ into the open set $\Pi$ of $\Psi$ bounded by $r_{1}$ and $r_{2}$. Since all geodesics are of class $A, r_{3}$ cannot cross $r_{1}$ or $r_{2}$ in any other point than $P$ and $r_{3}$ must have $A$ as point at infinity. It follows that $r_{3}$ is either part of a periodic geodesic $g$ or is asymptotic to a periodic geodesic $g$. Since $r_{3}$ cannot be asymptotic to either $r_{1}$ or $r_{2}$ (cf. Morse [1, Theorem 6]) $g$ must have on it points in the domain $\Pi$. But $g$ must emerge from $\Pi$ and hence must cross either $r_{1}$ or $r_{2}$. Suppose that $g$ and $r_{1}$ cross. If $g_{1}$ is the unending geodesic of which $r_{1}$ is part and if $g_{1}$ is periodic, then $g$ and $g_{1}$ are class $A$ periodic geodesics of the same type which cross and this is impossible (cf. Morse [1, Theorem 9]). If $g_{1}$ is not periodic it is asymptotic, when traced out in the sense approaching $A$, to a class $A$ periodic geodesic $b$ which is of the type of $g$. But now we have a class $A$ geodesic $g_{1}$ which is asymptotic to a class $A$ peri- 
odic geodesic $b$ and crosses a class $A$ periodic geodesic $g$ which is of the type of $b$. This is likewise impossible (cf. Morse [1, Lemma 11]).

Similar arguments hold if $g$ crosses $\boldsymbol{r}_{\mathbf{2}}$. The proof of the lemma is complete.

THEOREM 12.1. Two geodesic rays on an $M(f$, I, II) with the same initial point in $\Psi$ cannot be of the same type.

For suppose the theorem is not true. There would then be a point $P$ of $\Psi$ with two directed geodesic rays $r_{1}$ and $r_{2}$ with initial point $P$ and of the same type. Since $r_{1}$ and $r_{2}$ are of the same type, they have the same point at infinity $A$. Let $g_{1}$ and $g_{2}$ be the directed geodesics in $\Psi$ of which $r_{1}$ and $r_{2}$, respectively, are rays. Let $B_{1}$ and $B_{2}$ be the points at infinity, different from $A$, of the geodesics $g_{1}$ and $g_{2}$, respectively. We do not assume that $B_{1}$ and $B_{2}$ are necessarily distinct.

Since all yeodesics are of class $A$, the rays $r_{1}$ and $r_{2}$ together with their common point at infinity $A$ form a Jordan curve which bounds a region $\kappa^{\prime}$ of $\Psi$. Similarly, the rays $P B_{1}$ and $P B_{2}$ of $g_{1}$ and $g_{2}$, respectively, and the arc $B_{1} B_{2}$ of $U$ which does not contain $A$ bound a region $\kappa$ of $\Psi$. Let $Q$ be any point of $\kappa$ and $Q^{\prime}$ any point of $\kappa^{\prime}$. The unique geodesic segment $Q Q^{\prime}$ must cross both $g_{1}$ and $g_{2}$. It follows that the directed unending geodesic $g\left(Q, Q^{\prime}\right)$ of which the directed geodesic segment $Q Q^{\prime}$ is part must have $A$ as one of its points at infinity and its other point at infinity is in the $\operatorname{arc} B_{1} B_{2}$. Let $J$ denote the totality of directed geodesics $g\left(Q, Q^{\prime}\right), Q$ in $\kappa, Q^{\prime}$ in $\kappa^{\prime}$.

In the space $\Omega$ corresponding to the manifold obtained by identifying congruent points of $M(f$, I, II $)$ under the group $G$, the set $J$ determines an open set $J^{*}$. But an open set is a measurable set of positive measure and it follows from the hypothesis that $M(f, \mathrm{I}, \mathrm{II})$ is Poisson stable that the set $J^{*}$ contains a motion $m$ which is stable in the sense of Poisson. Let $g$ be a directed geodesic in the set $J$ such that $g$ represents $m$. Then $A$ is one of the points at infinity of $g$ and the other is some point $B$ of the arc $B_{1} B_{2}$. Let $Q$ be a point of $\kappa$ on $g$, let $r$ denote the directed geodesic ray $Q A$ which is part of $g$ and let $e$ be the initial element of $r$. It follows from the Poisson stability of $m$ that there is a sequence of elements $e_{1}, e_{2}, \cdots$ on $r$ such that if $N_{i}$ is the point bearing $e_{i}$, then $\lim _{i \rightarrow+\infty} N_{i}=A$ (in the euclidean sense) and there exist elements $e_{1}^{\prime}, e_{2}^{\prime}, \cdots$, respectively congruent to $e_{1}, e_{2}, \cdots$, such that $\lim _{n \rightarrow+\infty} e_{n}^{\prime}=e$.

Let $T_{n}$ be the transformation of $G$ such that $T_{n}\left(e_{n}\right)=e_{n}^{\prime}$. Since the set $J^{*}$ is open, there exists an integer $K$ such that $e_{n}^{\prime}$ determines a point in $J^{*}$ if $n>K$. Since all the directed geodesic rays with initial element determining a point in the set $J^{*}$ can be represented in $\Psi$ by rays with $A$ as point at infinity, we infer that $T_{n}(A)=A, n>K$. We evidently have $\lim _{n \rightarrow+\infty} T_{n}(Q)=B$ (in the euclidean sense).

Since $T_{n}(A)=A, n>K, T_{n}$ for $n>K$ is either: (1) a hyperbolic transformation with $A$ as one of its fixed points; (2) a parabolic transformation with $A$ as fixed point; (3) an inversion in a circle orthogonal to $U$ and inter- 
secting $U$ in $A$; or (4) an inverse conformal transformation with $A$ as one of its fixed points, the square of which is a hyperbolic transformation. But for $n$ large, $T_{n}(Q)$ is near $B$ and it is evident that (2) and (3) are excluded for $n$ sufficiently large. Thus there exists a hyperbolic transformation $T$ of $G$ with $A$ as one of its fixed points. According to Lemma 12.1 this is impossible if the rays $r_{1}$ and $r_{2}$ exist.

The proof of Theorem 12.1 is complete.

13. Existence of transitive geodesics. A directed unending geodesic (directed geodesic ray) on $M(f, G)$ is termed transitive if the elements on the geodesic (geodesic ray) form a set which is everywhere dense in the space $\Omega$ of elements on $M(f, G)$.

With a manifold $M(f, \mathrm{I}, \mathrm{II})$ there is associated a group $G$ and a manifold $M(f, G)$ obtained from $M(f$, I, II $)$ by identification of congruent points. A directed unending geodesic (directed geodesic ray) on $M(f$, I, II) is termed transitive if the corresponding geodesic (geodesic ray) on $M(f, G)$ is transitive. A necessary and sufficient condition that the directed geodesic $g$ (geodesic ray $r)$ on $M(f, \mathrm{I}, \mathrm{II})$ be transitive is that the totality of elements on $g(r)$ and all its congruent copies form a set which is everywhere dense in the set $E$.

LeMma 13.1. If there exists a point $P$ of $\Psi$ such that for $I$, an arbitrary interval of $U$, the totality of elements on all the geodesic rays of $M(f, I, I I)$ from $P$ to points of $I$ and on all copies of these rays forms a set which is dense in $E$, there exists a transitive geodesic ray from $P$ to some point of $I$.

The space $E$ contains a fundamental sequence $V_{1}, V_{2}, \cdots$ of neighborhoods such that any neighborhood of any point of $E$ contains a neighborhood of this sequence. It follows from the hypothesis of the lemma that there must exist a closed interval $I_{1}$ of $I$ such that any geodesic ray $r_{1}$ from $P$ to $I_{1}$ either has on it, or one of the copies of $r_{1}$ has on it, an element in $V_{1}$. There exists a closed subinterval $I_{2}$ of $I_{1}$ which has analogous properties with respect to $V_{2}$. Continuing thus, we obtain a sequence of closed intervals $I_{1} \supset I_{2} \supset I_{3} \supset \ldots$ and these must have a point $A$ in common. The geodesic ray from $P$ to $A$ is evidently transitive.

Lемма 13.2. The group $G$ corresponding to an $M(f, \mathrm{I}, \mathrm{II})$ is of the first kind.

The group $G$ is either of the first or second kind. If $G$ is of the second kind it is known (cf. Ford [1, p. 73]) that the fundamental region of $G$ abuts on the unit circle $U$ in at least one interval $I$. But any geodesic on $M(f, G)$ which is represented in $\Psi$ by a geodesic with a point at infinity in the interior of $I$ is not stable in the sense of Poisson. Since the set of elements on all such directed geodesics forms an open set in $\Omega$ we conclude that $M(f, \mathrm{I}, \mathrm{II})$ is not Poisson stable, contrary to hypothesis.

THEOREM 13.1. There exist transitive directed geodesic rays on any $M(f, \mathrm{I}, \mathrm{II})$. 
The proof of this theorem is similar to that of Theorem 3.1 of Hedlund [2]. We include a proof here for completeness.

It follows from Lemma 13.1 that it is sufficient to show that, if $P$ is a point of $\Psi$ and $I$ is an arbitrary interval of $U$, then the set $e(P, I)$ consisting of the elements on all the geodesic rays from $P$ to $I$ and on all their copies is everywhere dense in $E$.

Let $e(x, y, \phi)$ be an arbitrary point of $E$. The element $e$ determines a directed geodesic $g$, namely, that directed geodesic which passes through $S(x, y)$ the point bearing $e$ and has the direction $\phi$ at $S$. Let $A$ be the initial point at infinity of $g, B$ the terminal point at infinity of $g$, and $h$ the directed $H$-line $A B$. It follows from Theorem 9.2 that there is a point $S^{\prime}$ on $h$ such that $D\left(S, S^{\prime}\right)$ is not greater than the constant $R$.

According to known results (cf. Koebe [1, (II), p. 349]) concerning the existence of transitive hyperbolic rays, it follows from the fact that $G$ is of the first kind (Lemma 13.2) that there exists a directed hyperbolic ray $P C$, $C$ an interior point of $I$, such that the directed hyperbolic ray $P C$ is transitive. That is, the elements on $P C$ together with the elements on all copies of $P C$ form a set which is everywhere dense in $E$. It follows that there exists a sequence $T_{i}, i=1,2, \cdots$, of transformations of $G$ such that (in the euclidean sense) $\lim _{n \rightarrow+\infty} T_{n}(P)=A$ and $\lim _{n \rightarrow+\infty} T_{n}(C)=B$. Let $P_{n}=T_{n}(P)$ and consider the sequence of directed geodesic segments $P_{n} S$. With increasing $n$ the direction of $P_{n} S$ at $S$ must approach that of $g$ at $S$. For otherwise a subsequence of the set of geodesic segments $P_{n} S, n=1,2, \cdots$, could be chosen such that this subsequence has as limiting geodesic ray a geodesic ray $r$ with finite end point $S$, with point at infinity $A$, and such that $r$ is not identical with the part $A S$ of $g$. According to Theorem 12.1 this cannot be the case. Thus, if $e_{n}$ is the element of $P_{n} S$ at $S$, we infer that $\lim _{n \rightarrow+\infty} e_{n}=e$.

To complete the proof of the theorem it remains to show that for $n$ sufficiently large, there is an element $e_{n}^{\prime}$, congruent to $e_{n}$, in the set $e(P, I)$. The points $T_{n}^{-1}\left(S^{\prime}\right)$ have the property that (in the euclidean sense) $\lim _{n \rightarrow+\infty} T_{n}^{-1}\left(S^{\prime}\right)=C$. The point $T_{n}^{-1}(S)$ is at a distance not exceeding $R$ from $T_{n}^{-1}\left(S^{\prime}\right)$ and hence (in the euclidean sense) $\lim _{n \rightarrow+\infty} T_{n}^{-1}(S)=C$. There exists an $N$ such that for $n>N$, all the points $T_{n}^{-1}(S)$ lie in the domain bounded by $I$ and the geodesic rays from $P$ to the end points of $I$. But then $T_{n}^{-1}\left(P_{n} S\right)$, $n>N$, is a segment of one of the geodesic rays from $P$ to $I$, and if we let $e_{n}^{\prime}=T_{n}^{-1}\left(e_{n}\right)$, the element $e_{n}^{\prime}$ is congruent to $e_{n}$ and in the set $e(P, I)$.

The proof of the theorem is complete.

A directed geodesic $g$ on a two-dimensional Riemannian manifold $M$ is transitive, if the elements on $g$ form a set which is everywhere dense among the totality of elements on $M$.

COROLlaRy 13.1. There exist transitive geodesics on any closed orientable two-dimensional Riemannian manifold $M$ of class $C^{3}$ and of genus greater than 
one provided no geodesic on $M$ has on it two mutually conjugate points. There exist transitive geodesics on any closed non-orientable two-dimensional Riemannian manifold $M$ of class $C^{3}$ and of genus greater than two provided no geodesic on $M$ has on it two mutually conjugate points.

In either case, the universal covering surface of $M$ can be mapped conformally onto the interior $\Psi$ of the unit circle, thus determining a manifold $M(f, \mathrm{I}, \mathrm{II})$ and a group $G$. The manifold $M$ is obtained by identification of points which are congruent under $G$ and the stated corollary follows from Theorem 13.1.

\section{BibliograPHY}

O. BolzA

1. Vorlesungen über Variationsrechnung, Berlin, 1909.

C. CARATh ÉODORY

1. Über den Wiederkehrsatz von Poincaré, Sitzungsberichte der Preussischen Akademie der Wissenschaften, 1919, pp. 580-584.

L. R. FORD

1. Automorphic Functions, New York, 1929.

F. HAUSDORFF

1. Mengenlehre, Leipzig, 1927.

G. A. HedLund

1. Geodesics on a two-dimensional Riemannian manifold with periodic coefficients, Annals of Mathematics, (2), vol. 33 (1932), pp. 719-739.

2. Two-dimensional manifolds and transitivity, Annals of Mathematics, (2), vol. 37 (1936), pp. 534-542.

3. The dynamics of geodesic flows, Bulletin of the American Mathematical Society, vol. 45 (1939), pp. 241-260.

E. HopF

1. Zwei Sätze über den wairscheinlichen Verlauf der Bewegungen dynamischer Systeme. Mathematische Annalen, vol. 103 (1930), pp. 710-719.

2. Statistik der geodätischen Linien in Mannigfaltigkeiten negativer Krümmung, Berichten der Sächsischen Akademie der Wissenschaften zu Leipzig, 1939, pp. 261-304.

H. Hopf AND W. RINOW

1. Ueber den Begriff der vollständigen differentialgeometrischen Fläche, Commentarii Mathematici Helvetici, vol. 3 (1931), pp. 209-225.

P. KOEBE

1. Riemannsche Mannigfaltigkeiten und nicht euklidische Raumformen, Sitzungsberichte der Preussischen Akademie der Wissenschaften, (I), 1927, pp. 164-196; (II), 1928, pp. 345442.

M. MORSE

1. A fundamental class of geodesics on any closed surface of genus greater than one, these Transactions, vol. 26 (1924), pp. 25-60.

2. Instability and transitivity, Journal de Mathématiques Pures et Appliquées, (9), vol. 14 (1935), pp. 49-71.

S. B. MYERS

1. Riemannian manifolds in the large, Duke Mathematical Journal, vol. 1 (1935), pp. 39-49.

H. Poincaré

1. Les Méthodes Nouvelles de la Mécanique Céleste, vol. 3, Paris, 1899. 
W. Rinow

1. See H. Hopf.

2. Ueber Zusammenhänge zwischen der Differentialgeometrie im Grossen und im Kleinen, Mathematische Zeitschrift, vol. 35 (1932), p. 522.

H. Seifert and W. Threlfall

1. Lehrbuch der Topologie, Leipzig, 1934.

INSTITUTE FOR ADVANCED STUdy,

Princeton, N. J.

UNIVERSITY OF VIRGINIA,

Charlottesville, Va. 